\title{
Introduction to the RE'11 special issue: requirements in motion
}

\author{
Patrick Heymans
}

Published online: 15 May 2012

(C) Springer-Verlag London Limited 2012

In 2002, the IEEE Symposium on Requirements Engineering (created in 1993) and the Conference on Requirements Engineering (created in 1994) were merged into a single conference series to form the IEEE International Requirements Engineering Conference, which is commonly referred to as RE. Ever since then, RE is premier forum for researchers and practitioners to exchange on the latest developments in the field of Requirements Engineering.

It has now become a tradition that the best research papers of each yearly edition of RE are identified by the programme committee, and their authors are invited to submit and extended version for a special issue of the Requirements Engineering Journal (REJ), the leading specialized archival publication in the field.

As the Program Chair of the 19th IEEE International Requirements Engineering Conference (RE'11), it is my privilege and pleasure to introduce this special issue of the REJ which features extended versions of four distinguished papers from RE' 11 .

RE'11 was held in the beautiful medieval town of Trento, Italy, from August 29 to September 2, 2011. The main conference attracted 230 delegates from all over the world. It was preceded by 2 days of tutorials and workshops as well as the RE doctoral symposium. This year, we had 12 workshops and two co-located 2-day events, namely FMICS'11 (the 16th International Workshop on Formal Methods for Industrial Critical Systems) and IStar'11 (the 5 th International $i^{*}$ Workshop). The total number of attendants, including all co-located events, amounts to 300 .

P. Heymans $(\bowtie)$

PReCISE Research Centre, University of Namur (FUNDP),

Namur, Belgium

e-mail: patrick.heymans@fundp.ac.be
Overall, these statistics are excellent and demonstrate the healthy status of the RE community.

The special theme for this edition of the conference was "Requirements in Motion." As systems grow ever more complex, are developed globally, and become more interconnected, the impacts of changes are harder to analyse and control. In these settings, ensuring that software will continue to exhibit its intended properties becomes trickier. Efficient management of change in the broad diversity of RE contexts and across the whole RE process continuously calls for novel research ideas. For this reason, RE'11 specifically encouraged submissions that address requirements change and evolution.

This year, the research track received 138 submissions, from which we accepted 23 full-length papers, resulting in an acceptance rate of $16,7 \%$. The papers were selected using the multi-tiered blind review process developed over the years. Each paper was carefully reviewed by at least three program committee members. All papers with at least one positive review were then discussed by program committee and program board members using Cyberchair. Approximately one-third of the submissions eventually made it to the program board meeting in Namur, Belgium, where the final decisions were made. The 23 research papers were divided into eight paper sessions which showcased ground-breaking research on a rich variety of topics. Two of these sessions were devoted to the special theme.

Out of these 23 already highly select papers, the program committee nominated one best paper and five distinguished papers. Their authors were invited to submit a revised and extended version for possible inclusion in the REJ. Four of these papers eventually made it to this special issue after going through a standard journal blind review process with subsequent revisions. Part of the referees were 
kept from the RE'11 program committee, and part were external reviewers. The four papers are an excellent sample of the best contemporary research in RE.

The first paper accepted to this special issue of the REJ, entitled 'Power and Politics in Requirements Engineering: Embracing the Dark Side?", by Milne and Maiden, is an expanded version of the RE'11 best paper award winner. The paper addresses a long overdue topic: the role of power and politics in RE. It stems from the observation that sociotechnical systems are becoming increasingly more complex, uncertain and organizationally embedded and that power and politics are therefore ever more relevant to RE. The authors open up the debate and propose a framework to understand the networks of power relationships that are at play in RE projects. The paper features a case study that demonstrates how the framework could become a new precious tool for practitioners.

The second paper also deals with an increasingly important concern in RE: compliance of software with laws and regulations. "A Legal Cross-References Taxonomy for Reasoning about Compliance Requirements" by Maxwell, Anton, Swire, Riaz and McCraw is the most recent in a series of papers from Anton et al. that propose and evaluate solutions for ensuring legal compliance of requirements. Cross-references within and across laws/regulations may cause problems by introducing conflicts and ambiguities. In this paper, the authors perform a multi case study from which they derive a taxonomy of legal cross-references. This taxonomy can be used by engineers to better assess the effects of cross-references on compliance requirements and, ultimately, to resolve conflicts between them.

The two remaining papers address the special theme of the conference more prominently.

The third paper is "Early Failure Prediction in Feature Request Management Systems: An Extended Study" by Fitzgerald, Letier and Finkelstein. Online feature request management systems are becoming popular tools to handle change requests from end-users. However, a problem is to estimate how much analysis effort must be spent on each feature request. In this paper, the authors have studied feature request failures in seven large projects. They have developed a tool-supported framework for constructing failure prediction models and compared prediction techniques for these projects. The study provides evidence that early failure prediction can indeed help project managers make better informed decisions on how and how much analysis to perform on incoming requests.

This special edition concludes with the paper "An Investigation of the Causes and Effects of Software Requirements Change: Two Case Studies" by McGee and Greer. Their first case study seeks to understand how different categories of requirements changes can be an effective basis for change classification and measurement.
Significant differences in cost, customer value and impact on management have been highlighted. In the second case study, the authors identify a consistent correlation between requirements dependencies and volatility. They also observe that different change sources affect different groups of requirements. Overall, their taxonomy appears to be a very promising tool to better anticipate and manage requirements changes.

In addition to thanking the authors of these papers for their outstanding work, I would like to also congratulate the recipients of the 10-year impact award. Whereas distinguished papers are selected based on the quality and potential of their content, impact award laureates are recognized for the actual impact that their work has had over the past decade. This year, the award went to Axel van Lamsweerde for his 2001 mini-tutorial entitled "GoalOriented Requirements Engineering: A Guided Tour." With over 1000 citations counted by Google Scholar at the time of writing this editorial, van Lamsweerde's tutorial is by an order of magnitude the most cited paper from RE' 01 . More importantly, this paper was the first to suggest "what a [comprehensive] goal-oriented requirements engineering method may look like." This was illustrated through a case study of a safety-critical train control system. The paper also contained an insightful introduction to goal-orientation as well as a comparative review of existing work in the area. Additionally, it reported experience in applying the KAOS goal-oriented method and discussed tool support. This work has been inspiring to a whole generation of RE researchers and could be seen as an early preview of the long-awaited book that van Lamsweerde published in 2009. Two papers from RE'01 received honorable mentions for the 10-year impact award. They are "Model Checking Early Requirements Specifications in Tropos" by Fuxman, Pistore, Mylopoulos and Traverso; and "An Industrial Survey of Requirements Interdependencies in Software Product Release Planning" by Carlshamare, Sandahl, Lindvall, Regnell and Natt och Dag. The former was one of the very first concrete attempts to apply model checking to requirements models; the latter delivered an enlightening survey of current practice, a type of paper that became much more common over the past decade, but which back in 2001 was very unusual.

RE'11 welcomed keynotes from three outstanding speakers. Joanne Atlee (University of Waterloo), a wellknown member of the RE community and former programme chair of RE'05, shed light on one of the most challenging issues related to system evolution: feature interaction. Michael Whalen (University of Minnesota) reported experience in applying model checking techniques on industrial safety-critical systems, again demonstrating that good requirements are key to preventing failure. Finally, Cecilia Mascolo, reader at the University of 
Cambridge and a renowned mobile systems researcher, told us a fascinating tale of cutting-edge multi-disciplinary research involving computer scientists, zoologists and social psychologists, emphasizing the ubiquity of requirements for systems that utilize mobile and sensing technologies.

In addition to keynotes, the program included two minitutorials: Alistair Sutcliffe (University of Manchester) introduced the audience to "Emotional Requirements Engineering" whereas Björn Regnell (Lund University) presented and reflected on the challenges of "Large-scale Feature Evolution" in the mobile domain. Last but not least, the RE' 11 program featured an industry track chaired by Samuel Fricker, two panels (respectively, on tacit knowledge and design requirements), a fun and highly interactive technology transfer session, as well as the usual posters and demo session.

Putting together this special issue is a welcome privilege of the RE'11 Program Chair. However, this issue would not exist without the considerable efforts of a highly effective and motivated team. I would like to thank John Mylopoulos, General Chair of RE'11, Anna Perini and Angelo
Susi, Organization Chairs, who hosted the conference at the University of Trento, and Samuel Fricker, Industry Chair. I would also like to thank Neil Maiden, the current chair of the RE steering committee, his predecessor Martin Glinz, as well as Peri Loucopoulos, Bill Robinson, and the REJ editorial team for their ongoing support. I extend my gratitude to former RE Program Chairs, Jane Cleland-Huang, Kevin Ryan and Alistair Sutcliffe, who have provided guidance and advice whenever I needed it. Finally, I would like to thank the members of the RE' 11 organizing committee, program committee and program board, as well as the additional anonymous referees who reviewed the invited REJ submissions.

I am confident that the contents of this special issue will inspire junior and experienced RE researchers alike. I invite all of them as well as anybody else who is interested in $\mathrm{RE}$ to participate in the forthcoming RE conferences: RE' 12 to be held in Chicago, USA, and RE' 13 to be held in Rio de Janeiro, Brazil.

Patrick Heymans

Program Chair of RE'11 and Special Issue Editor. 